\title{
Citrus reticulata CrRAP2.2 Transcriptional Factor Shares Similar Functions to the Arabidopsis Homolog and Increases Resistance to Xylella fastidiosa
}

\author{
Willian Pereira, ${ }^{1,2,3}$ Marco Takita, ${ }^{1}$ Maeli Melotto, ${ }^{3}$ and Alessandra de Souza ${ }^{1,+}$ \\ ${ }^{1}$ Centro de Citricultura Sylvio Moreira (CCSM/IAC), Cordeirópolis, São Paulo 13490-970, Brazil \\ ${ }^{2}$ Universidade Estadual de Campinas (UNICAMP), Campinas, São Paulo 13083-862, Brazil \\ ${ }^{3}$ Department of Plant Sciences, University of California, Davis, CA 95616, U.S.A.
}

Accepted 16 December 2019.

\begin{abstract}
Xylella fastidiosa is a worldwide multihost pathogen that causes diseases in different crops. It is considered a new global threat and substantial efforts have been made in order to identify sources of resistance. Indeed, many genes have been associated with resistance to $X$. fastidiosa, but without functional validation. Here, we describe a $C$. reticulata gene homologous to the transcriptional factor RAP2.2 from Arabidopsis thaliana that increases resistance to citrus variegated chlorosis (CVC). This gene was previously detected in $C$. reticulata challenged with $X$. fastidiosa. Bioinformatics analysis together with subcellular localization and auto-activation assays indicated that $R A P 2.2$ from $C$. reticulata $(C r R A P 2.2)$ is a transcriptional factor orthologous to AtRAP2.2. Thus, we used $A$. thaliana as a model host to evaluate the functional role of $\mathrm{CrRAP2.2}$ in $X$. fastidiosa resistance. The inoculation of $X$. fastidiosa in the $A$. thaliana rap2.2 mutant resulted in a larger bacterial population, which was complemented by $C r R A P 2.2$. In addition, symptoms of anthocyanin accumulation were higher in the mutant, whose

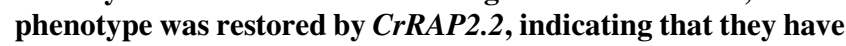
conserved functions in plant defense response. We therefore transformed $C$. sinensis with $C r R A P 2.2$ and verified a positive correlation between CVC resistance and gene expression in transgenic lines. This is the first study using $A$. thaliana as model host that characterizes the function of a gene related to $X$. fastidiosa defense response and its application in genetic engineering to obtain citrus resistance to $\mathrm{CVC}$.
\end{abstract}

Keywords: Citrus reticulata (mandarin), Citrus sinensis (sweet orange), citrus transgenic plants, CVC resistance, RAP2.2 transcriptional factor, Xylella-Arabidopsis interaction

${ }^{\dagger}$ Corresponding author: Alessandra A. de Souza; desouza@ccsm.br

Funding: This research was funded by Fundação de Amparo à Pesquisa do Estado de São Paulo grants 2013/10957-0, 2013/26944-5, and 2015/504626, Coordenação de Aperfeiçoamento de Pessoal de Nível Superior grant 001. and Conselho Nacional de Desenvolvimento Científico e Tecnológico grants 202762/2015-7 and 405004/2013-3.

*The $\boldsymbol{e}$-Xtra logo stands for "electronic extra" and indicates that four supplementary figures and one supplementary table are published online.

The author(s) declare no conflict of interest.

๑) 2020 The American Phytopathological Society
Xylella fastidiosa is a xylem-limited phytopathogen that causes diseases in many perennial crops such as citrus, grape, almond, coffee, and plum (Rapicavoli et al. 2018a). Until recently, $X$. fastidiosa was found only in the Americas; however, in 2013 it appeared in southern Italy causing olive quick decline syndrome (OQDS) in olive trees (Saponari et al. 2013). Since then, other European countries have reported the bacterium, and it has rapidly become a major concern in regard to food security. Moreover, the bacterium has been categorized as a quarantine pathogen included in the European and Mediterranean Plant Protection Organization (EPPO) A2 list (available online). Currently $X$. fastidiosa was divided into three subspecies: subsp. fastidiosa, pauca, and multiplex (Marcelletti and Scortichini 2016). X. fastidiosa subsp. pauca is responsible for causing coffee leaf scorch, citrus variegated chlorosis (CVC), and OQDS. In citrus, X. fastidiosa affects all varieties of sweet orange (Citrus sinensis L. Osbeck) but no other Citrus species, including mandarins (Citrus reticulata Blanco) and their hybrids (Coletta-Filho et al. 2007; Niza et al. 2015).

Many studies have assessed the genetic mechanism underlying the defense response of $C$. reticulata to $X$. fastidiosa through global gene expression analysis (de Souza et al. 2007a; Mauricio et al. 2019; Rodrigues et al. 2013). The genetic mechanism related to $C$. reticulata resistance involves the recognition of $X$. fastidios $a$ by specific receptors and the triggering of genes involved with cell-wall modification and activation of hormone pathways (Niza et al. 2015; Rodrigues et al. 2013). Similarly, the recognition of receptors and cell-wall modification have also been shown as mechanisms of $X$. fastidiosa resistance in a tolerant olive cultivar (Giampetruzzi et al. 2016; Sabella et al. 2018). Gene expression analyses of citrus and grape at different timepoints of $X$. fastidiosa colonization showed that different genes are expressed in both hosts in early and late stages of infection. These differences seem to be a consequence of phytohormone signaling pathways, which are differentially regulated according to the stage of X. fastidiosa infection (Rapicavoli et al. 2018b; Rodrigues et al. 2013; Zaini et al. 2018). In citrus and grape resistant to $X$. fastidiosa, defense response involves the participation of salicylic acid-mediated defense pathways, which seem to be inhibited in grape and citrus susceptible to $X$. fastidiosa infection (de Souza et al. 2007b; Rapicavoli et al. 2018b; Rodrigues et al. 2013; Zaini et al. 2018). Despite abundant research on the genes related to the defense response against $X$. fastidiosa in different hosts, the functional role of such genes in the mechanism of resistance remains unknown in $X$. fastidiosa-plant host interactions. 
Gene functional analysis involving sweet orange is very time-consuming due to slow growth of the perennial host, long juvenile period, and long incubation before the development of symptoms (Almeida et al. 2001; Coletta-Filho et al. 2007; Peña et al. 2001). However, studies have developed methods to overcome this issue; specifically, Arabidopsis thaliana is a good alternative host that can be used to accelerate functional studies regarding the genetic mechanism associated with X. fastidiosa-plant interactions (Pereira et al. 2019; Rogers 2012). Genes encoding putative transcription factors of the APETALA2/ETHYLENE RESPONSIVE FACTOR (AP2/ERF) family were identified as candidate genes conferring resistance of $C$. reticulate to $X$. fastidiosa infection (Rodrigues et al. 2013). However, they have not yet been functionally characterized.

Thus, in this study, we sought to analyze the molecular function of this gene, hereafter named $C r R A P 2.2$. We found that $C r R A P 2.2$ is highly similar to the Arabidopsis AtRAP2.2 that is associated with resistance to the pathogen Botrytis cinerea (Zhao et al. 2012). Using a combination of bioinformatics, genetic, and phenotypic analyses, we determined that ectopic expression of $C r R A P 2.2$ in the Arabidopsis rap2.2 mutant complements its susceptibility to $X$. fastidiosa in early stages of infection. Furthermore, expression of CrRAP2.2 in C. sinensis was positively correlated with CVC resistance. Our results suggest that AtRAP2.2 and CrRAP2.2 share similar functions as transcriptional factors and they act as positive regulators of immunity against $X$. fastidiosa in both genera Arabidopsis and Citrus. Thus, we used a RAP2.2 from $C$. reticulata to transform $C$. sinensis aiming to confer resistance to $X$. fastidiosa. These two species can naturally cross and are frequently used in breeding programs (Coletta Filho et al. 2007; Mauricio et al. 2019) but other traits are carried, altering commercial features. In this case, transgenic plants carrying genes from genetically compatible species might be an alternative for development of resistant plants without loss of the high-quality commercial features and consumer acceptance.

\section{RESULTS}

\section{Expression of $\operatorname{CrRAP2.2}$ is associated} with $X$. fastidiosa defense response.

Here, we compared the expression of RAP2.2 between susceptible species $C$. sinensis, and resistant species $C$. reticulata. Although

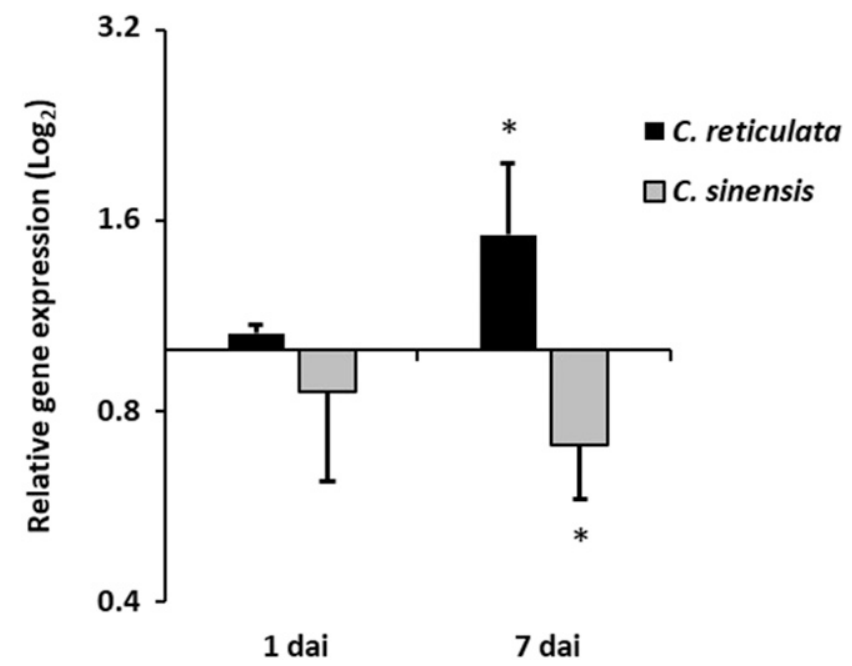

Fig. 1. Expression analysis of the citrus RAP2.2 in Citrus reticulata and $C$. sinensis after inoculation with Xylella fastidiosa. Gene expression in each species relative to the respective noninoculated plants (mock set to 1). Results are shown as the mean of triplicates. Asterisks indicate statistical significance using a Student's $t$ test $(P<0.05)$; dai $=$ days after inoculation. not statistically significant, the expression of $R A P 2.2$ is slightly induced 1 day after inoculation (dai) with $X$. fastidiosa in the resistant C. reticulata; the increase of expression of $R A P 2.2$ in $C$. reticulata was significant at 7 dai. Repression of $R A P 2.2$ was observed in the susceptible $C$. sinensis at the same timepoints (Fig. 1).

Alignment of the predicted RAP2.2 protein from C. reticulata (CrRAP2.2) and the RAP2.2 protein from Arabidopsis (AtRAP2.2), using BLASTP, revealed high similarity, with $46 \%$ amino acid identity over the entire sequence (Fig. 2A). Both proteins show highly conserved domains, particularly the APETALA2 domain and nuclear localization site (NLS) domain (Fig. 2A), which are also present in RAP2.2 (SUB1) from Oryza sativa (Hinz et al. 2010).

To structurally compare the APETALA2 domain of both proteins with three-dimensional (3D) protein structures, we looked for the highest match in the Protein Data Bank database, which was the GCC-box binding domain (GBD) of $A$. thaliana ethylene responsive element binding factor 1 (AtERF1) (Allen et al. 1998). Both proteins shared a high sequence identity $(72.41 \%)$ with the GBD, including all the residues responsible for DNA binding in the $\beta$-sheets (Fig. 2B).

\section{CrRAP2.2 is a transcription factor.}

Aiming to confirm that CrRAP2.2 is a transcription factor similar to AtRAP2.2, we evaluated its transcriptional activity using a yeast system. We observed that the full-length activation domain (AD)::CrRAP2.2 protein binds to and activates the lacZ promoter in Saccharomyces cerevisae EGY48 (p8op-lacZ) (Fig. 3A, indicated by the blue color in colony 3). Furthermore, we evaluated the three truncated versions of AD::CrRAP2.2 lacking amino acids (CrRAP2.2 $\Delta 1-230$, CrRAP2.2 $\Delta 230-388$, and CrRAP2.2A153-388), corresponding to colonies 4,5 , and 6 , respectively (Fig. 3A). Only CrRAP2.2 $\Delta 1-230$ was able to activate the lac $\mathrm{Z}$ promoter; therefore, the activation domain of the protein is located in its C-terminal region, which is in agreement with that observed for the AtRAP2.2 transcription factor (Welsch et al. 2007).

In addition, we analyzed the subcellular localization of CrRAP2.2, using a 35S promoter-driven green fluorescent protein (GFP)::CrRAP2.2 construct that was transiently expressed in $N$. benthamiana leaves, revealing that the GFP:: CrRAP2. 2 protein is exclusively localized in the nucleus of epidermal cells (Fig. 3B).

\section{AtRAP2.2 contributes to $X$. fastidiosa resistance} and ectopic expression of CrRAP2.2 complements rap2.2.

As AtRAP2.2 has been implicated in plant immune response (Zhao et al. 2012) and CrRAP2.2 seems to be an ortholog of AtRAP2.2, we performed a genetic complementation assay by expressing the CrRAP2.2 protein in the A. thaliana rap2.2 mutant to analyze its role in resistance to $X$. fastidiosa. Interestingly, we observed a significantly higher $X$. fastidiosa population (50-fold difference) in rap2.2 compared with the Col-0 wild type (Fig. 4). However, this difference is not present 2 weeks after inoculation, suggesting that one or more other genetic mechanisms could take place at later stages of infection. Nonetheless, this observation created an opportunity to test whether the early susceptibility of rap 2.2 could be rescued by CrRAP2.2 and whether CrRAP2.2 could enhance the resistance response in $A$. thaliana Col-0 at later stages (2 to 3 weeks) of $X$. fastidiosa infection. To test these hypotheses, we first created two independent transgenic lines of rap2.2 and Col-0 expressing p35S-CrRAP2.2, named rap2.2/CrRAP2.2 and Col-0/CrRAP2.2, respectively (Supplementary Fig. S3).

We found that expression of CrRAP2.2 in the rap2.2 background restored the $X$. fastidiosa colonization phenotype, as the bacterial population observed 1 week after inoculation was 
similar to that observed in Col-0 at the same timepoint (Fig. 4). These findings suggest that native AtRAP2.2 is involved in resistance to $X$. fastidios $a$ at the early stage of infection and CrRAP2.2 has a role similar to that of AtRAP2.2, indicating that these proteins are functional orthologs. Furthermore, expression of CrRAP2.2 in both rap2.2 and Col-0 backgrounds significantly increased the resistance of these plants toward $X$. fastidiosa, suggesting that CrRAP2.2 is a positive regulator

A

CrRAP2.2 1

1

2

$\square$ NLS

AP2

AtRAP2 21

MCGGAIISGYIPPTVPGRPKKLTAEYLWPELK--.-- - RSSNKKYSKPIVPDDFEADFQ MCGGAIIS +IPP $R++T$ E++WP+LK + SNK+ +DDDFEADFQ

AtRAP2.2 1 MCGGAIISDFIPPP---RSLRVTNEFIWPDLKNKVKASKKRSNKRSDFFDLPDDFEADFQ

CrRAP2.2 54

GFKDDE $\stackrel{* * * * * *}{*}$ * -SHGSTAVK GFKDD $S \mathrm{D}+\mathrm{D} D+{ }_{+}+\mathrm{KPF} F+A \mathrm{~K}++\mathrm{V}_{+}$

AtRAP2.2 58 GFKDD---SAFDCE---DDDDVFVNVKPFVFTATTKPVASAFVSTGIYLVGSAYAKKTVE

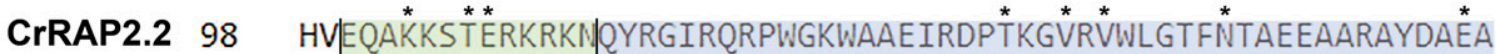
EQA+KS++RKRKN:YYRGIRQRPWGKWAAEIRDP KG R WLGTF+TAEEAARAYDA A AtRAP2.2 112 SAEQAEKSSKRKRKNQYRGIRQRPWGKWAAEIRDPRKGSREWLGTFDTAEEAARAYDAAA

CrRAP2.2 158

RRIRGKKKAKVNFPDEE - - -TPAAAPKSSVKANS-OKSV - PKACLSPVQPSLNQNFNYLNN RRIRG KAKVNFP+E + + + SKN+QKSV P ++ VQ + + YNN

AtRAP2.2 172 RRIRGTKAKVNFPEEKNPSVVSQKRPSAKTNNLQKSVAKPNKSVTLVQQPTHLSQQYCNN

CrRAP2.2 212

SDQDYYNNVSILEEKPQV - -NQYGYMGSFPDDGELGFKPFVPTADDTPVYFNSDQGSNSF $\mathrm{S}++++\mathrm{S}+\mathrm{EEKPQ}+\mathrm{NQ}+\mathrm{G}$ SF $\mathrm{G} \mathrm{G}++$ YF+SDQGSNSF

AtRAP2.2 232 SFDNSFGDMSFMEEKPQMYNNQFGLTNSFDAGGNNGYQ-

CrRAP2.2 270

DCSDFGWGEQAPKTPEISSVLEATPEVDESQFVDAVANPMKKLKSNSENAVPVQDNNGKS $\mathrm{DCS}+\mathrm{FGW}+\mathrm{PKTPEISS}+\mathrm{L}+\mathrm{E}+\mathrm{FV}+\mathrm{N}$ KKLK NS+

AtRAP2.2 281 DCSEFGWSDHGPKTPEISSMLVNN - - NEASFVEET-NAAKKLKPNSDE -

CrRAP2.2 330

LSDELLAF -DNQVKFLPMPYPEGSWEASLDAFLNGDNTQDGGNLMNLWSFDDFPSTLGGV

AtRAP2.2 326

$\mathrm{SD}+\mathrm{L}+\mathrm{A}+\mathrm{DN}+\mathrm{P}+$ EA L A G TQ+ N + LWS D+ L G

CrRAP2.2 389

$\begin{array}{llll}\text { AtRAP2.2 } & 379 & + & + \\ \text { F } & 379\end{array}$

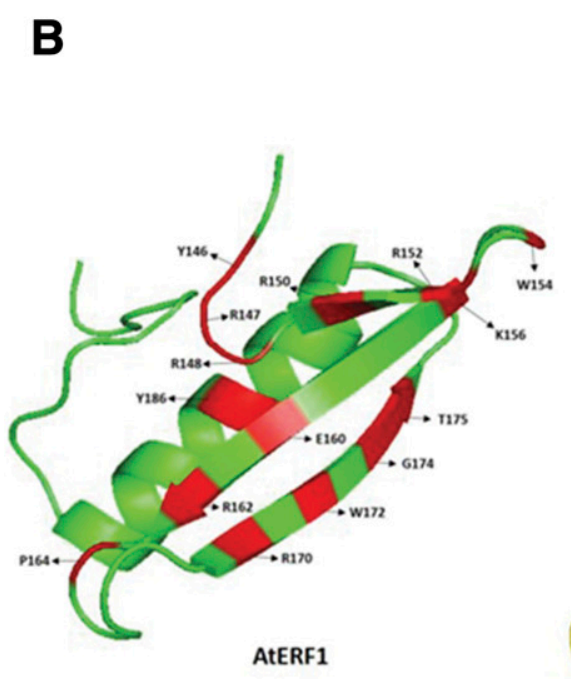

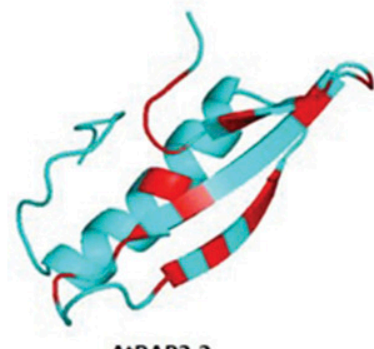

AtRAP2.2

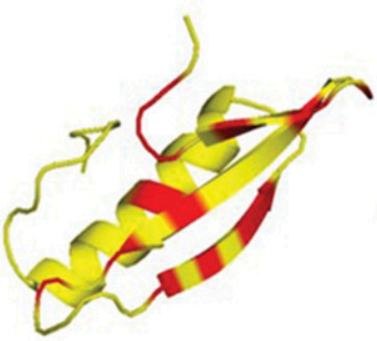

CrRAP2.2

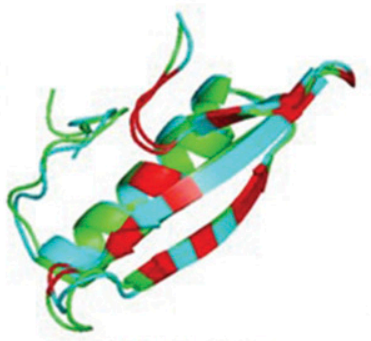

AtERF1+AtRAP2.2

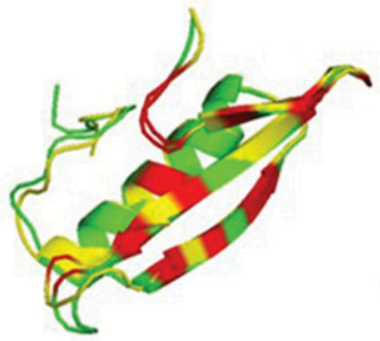

AtERF1+CrRAP2.2

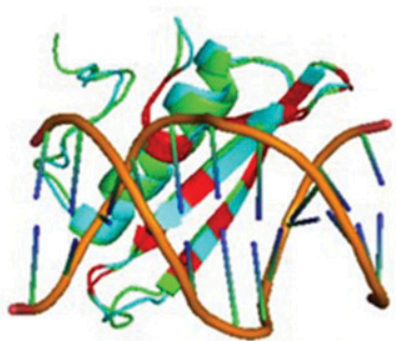

AtERF1+AtRAP2.2+DNA

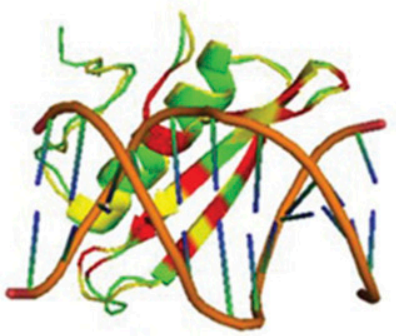

AtERF1+CrRAP2.2+DNA

Fig. 2. CrRAP2.2 is highly similar to the AtRAP2.2. A, Protein sequence alignment of CrRAP2.2 and AtRAP2.2 showing conserved regions: the N-terminal MCGG motif (1), an unknown function (2), nuclear localization signal (NLS), and APETALA2 (AP2) domains. CrRAP2.2 and AtRAP2.2 share 100\% identity in region $1,76.19 \%$ identity in region $2,76.92 \%$ identity in the NLS domain, and $88.33 \%$ identity in the AP2 domain. B, Three-dimensional protein structure alignment of the ethylene responsive element binding factor 1 (AtERF1) with AtRAP2.2 and CrRAP2.2, showing that both share the same amino acids required to bind DNA in the GCC-box binding domain (marked in red). AtERF1 structure is shown in green, AtRAP2.2 model in blue, and CrRAP2.2 model in yellow. Proteins are shown individually, merged, and merged with DNA binding regions. 
of plant immunity against this bacterium. In addition, the expression of CrRAP2.2 in Col-0, which carries the endogenous AtRAP2.2, does not lead to increased resistance as compared with rap2.2 plants (Fig. 4), indicating no synergism between the RAP2.2 protein from Arabidopsis and Citrus species. In fact, the enhanced resistance in Col-0/CrRAP2.2 plants, compared with Col-0 plants, indicates that CrRAP2.2 alone can be a strong positive regulator of immunity when constitutively expressed.

We recently demonstrated that anthocyanin accumulation is a useful phenotypic marker for $X$. fastidiosa colonization in Arabidopsis (Pereira et al. 2019). In agreement with the high colonization observed in rap2.2 at the early stage of infection (Fig. 4), a significant accumulation of anthocyanin was observed in the rap2.2 mutant compared with the wild-type plant infected with $X$. fastidiosa (Fig. 5). Furthermore, complementation of the rap2.2 mutant with CrRAP2.2 restored the Col0 wild-type phenotype; significantly lower anthocyanin accumulation was observed in Col-0 overexpressing $\mathrm{CrRAP} 2.2$ (Fig. 5). These results reinforce the association of high anthocyanin accumulation with increased $X$. fastidiosa colonization in A. thaliana and suggest that AtRAP2.2 is either directly or indirectly related to this phenotype.

\section{Transgenic sweet orange plants overexpressing $\mathrm{CrRAP2.2}$ increases resistance to $\mathrm{CVC}$.}

To study the potential role of CrRAP2.2 in conferring resistance to CVC, the A596p9ioGusi-FMV::CrRAP2.2 construction was used to transform sweet orange (Pineapple variety). We obtained six transgenic lines with variable expression levels of the transgene (Fig. 6A). The real time quantitative PCR (RT-qPCR) analysis indicated that transgenic line T142 had an expression level 64 times greater than the wild type, whereas the other lines had expression levels only 1.5 to four times greater than the wild type (Fig. 6A). Based on this result, we selected three lines with different transgene expression levels (T142, T168, and T172) to assess the severity of CVC symptoms.

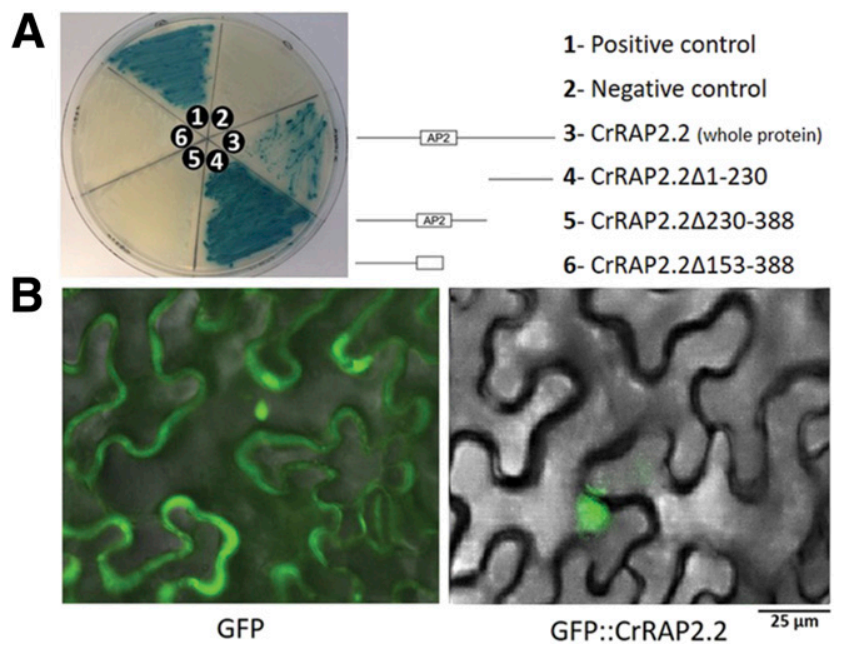

Fig. 3. Transcriptional activity assay and subcellular localization of the CrRAP2.2 protein. A, Transcriptional activity assay of the CrRAP2.2 protein in a yeast system. Each colony number corresponds to a different construct expressed in Saccharomyces cerevisae EGY48 (p8op-lacZ). The negative control is the yeast strain EGY48 (p8op-lacZ) and the positive control is the yeast strain EGY48 (p8op-lacZ) containing vector pLexApos. The size domains of CrRAP2.2 expressed in each construct is referred by the numbers 1 to 6 . B, Subcellular localization of CrRAP2.2 in Nicotiana benthamiana cells. The pB7WGF2 empty vector was used to show the localization of green fluorescent protein (GFP) alone.
The first symptoms of CVC were observed approximately 9 months after inoculation in the wild type and the T168 transgenic line. This line showed the lowest CrRAP2.2 expression level (only 1.5-fold higher than the wild type) among the transgenic lines. After 12 months, symptoms started to appear in T172, which has approximately threefold higher CrRAP2.2 expression. Line T142 was symptom-free until the end of the experiment (18 months after inoculation), when the wild type and T168 plants had severe CVC symptoms (Fig. 6B and C; Supplementary Fig. S4A). There is a good correlation between CrRAP2.2 expression and the severity of CVC; the greater the expression of $C r R A P 2.2$ (Fig. 6A) in the transgenic lines, the lower the severity of symptoms observed in C. sinensis plants (Fig. 6B and C).

\section{DISCUSSION}

Many genes have been identified as associated with resistance to X. fastidiosa (de Souza et al. 2007a; Giampetruzzi et al. 2016; Rapicavoli et al. 2018b; Rodrigues et al. 2013; Zaini et al. 2018), including a putative transcription factor of the AP2/ERF family in the early response of $C$. reticulata, a citrus species resistant to this bacterium (de Souza et al. 2007a; Rodrigues et al. 2013). In addition, this gene was also associated with early genetic response to $X$. fastidiosa in grapevine (Rapicavoli et al. 2018b). Different functions have been attributed to the AP2/ERF family of transcription factors, such as tolerance to abiotic (Xu et al. 2006, Hinz et al. 2010) and biotic stresses (Zhao et al. 2012).

Bioinformatics and experimental analyses indicated that the citrus gene CrRAP2.2 is an Arabidopsis RAP2.2 ortholog (Figs. 2 and 3 ). A higher number of $X$. fastidiosa cells was observed in the A. thaliana rap 2.2 mutant 1 week after inoculation, which was complemented with $C r R A P 2.2$. This finding reinforces the idea that RAP2.2 plays a role in the genetic mechanism of resistance at the beginning of the infection period. Similarities

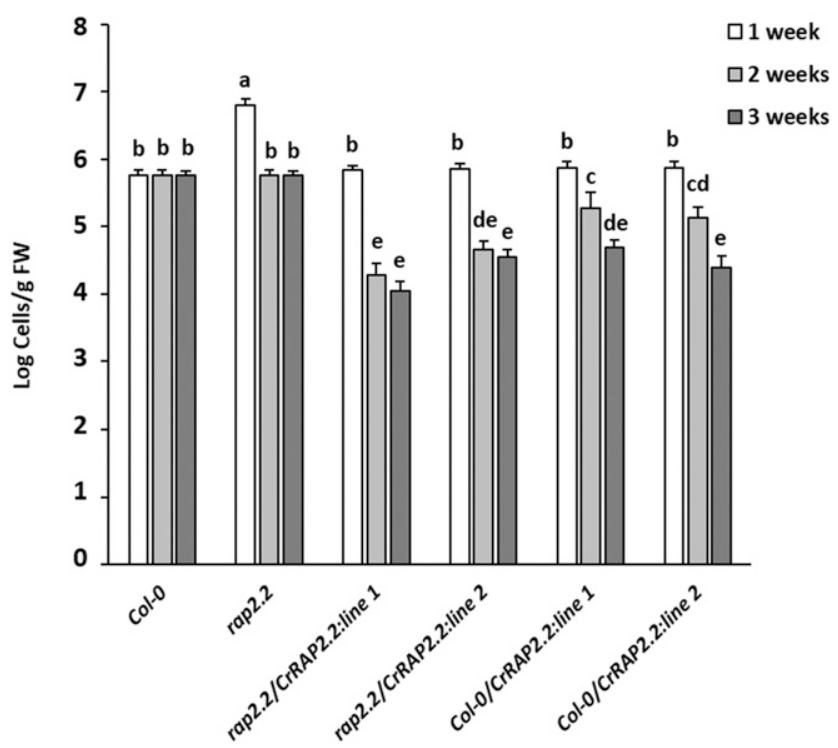

Fig. 4. Influence of RAP2.2 on Xylella fastidiosa colonization in Arabidopsis thaliana over the duration of the study. The $X$. fastidiosa population in the Col-0 wild type, rap2.2 mutant line, rap2.2/CrRAP2.2 complemented genotypes, and Col-0/CrRAP2.2 lines 1 and 5 overexpressing genotypes at 1,2 , and 3 weeks after inoculation. Results are shown as the mean of six replicates. Bars indicate standard errors. Different letters on top of the bars indicate statistically significant differences among the means calculated with a one-way analysis of variance, followed by a Tukey test $(P<0.05)$. $\mathrm{FW}=$ fresh weight. 
between $X$. fastidiosa and $B$. cinerea have also been demonstrated in grapevines (Agüero et al. 2005). Transgenic plants overexpressing polygalacturonase-inhibiting proteins that specifically inhibit fungal endo-polygalacturonases showed improved resistance to both $B$. cinerea and $X$. fastidiosa (Agüero et al. 2005). In agreement with these results, a recent study demonstrated that grapevine transgenic plants overexpressing a plant polygalacturonase inhibitory protein (PGIP) prevents Pierce's disease by inhibiting the breakdown of pectin present in primary cell walls (Dandekar et al. 2019). Therefore, the similarity of $X$. fastidios $a$ and $B$. cinerea is the ability to degrade cell wall and to trigger the plant immune response (Choi and Klessig 2016; Glazebrook 2005). In this context, the participation of the ethylene responsive factor RAP2.2 might be involved in the modification of cell walls, acting to impair $X$. fastidiosa colonization (Pérez-Donoso et al. 2007). In the resistant $C$. reticulata, the fortification of the cell wall due to an increase of lignin was attributed to be a genetic mechanism to avoid $X$. fastidiosa colonization, which is delayed in susceptible C. sinensis (Niza et al. 2015; Rodrigues et al. 2013). In olive, the greater increase in total lignin in the resistant cultivar Leccino, compared with the susceptible cultivar Cellina di Nardò, has an essential role in its tolerance against $X$. fastidiosa (Sabella et al. 2018).

The overexpression of CrRAP2.2 increased plant resistance in both rap2.2/CrRAP2.2 and Col-0/CrRAP2.2, verified by the lower bacterial population observed at 2 and 3 weeks after inoculation (Fig. 4). Regarding the later stages of infection (i.e., at 2 and 3 weeks) in A. thaliana (Pereira et al. 2019), other genetic mechanisms occur, which explains the similar bacterial population observed in the mutant and the wild type at these timepoints (Fig. 4). Different genetic defense responses have been observed in both citrus and grape at early and late stages of $X$. fastidiosa infection; specifically, different genes involved with modulation of hormone biosynthesis and signaling play a role at each of the different stages of infection. Nonetheless, the salicylic acid-mediated defense pathway seems to be essential in later stages of infection to contain bacterial spread (Rapicavoli et al. 2018b; Rodrigues et al. 2013; Zaini et al. 2018). The change in plant response could be associated with different bacterial lifestyles in the plant host, such as biofilm and

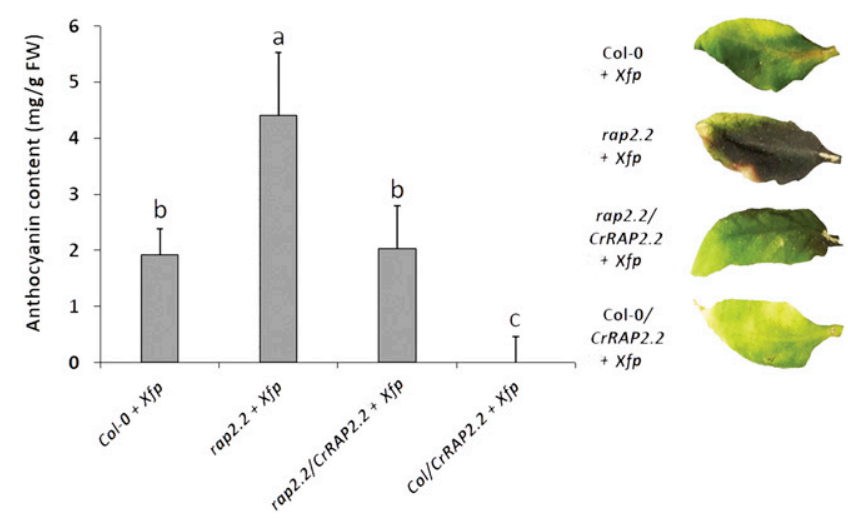

Fig. 5. Anthocyanin accumulation in Arabidopsis thaliana infected with Xylella fastidiosa. Anthocyanin was quantified in the Col-0 wild type, rap 2.2 mutant line 1 , rap2.2/CrRAP2.2 complemented line 1 , and Col0/CrRAP2.2 overexpressing line 1 at 3 weeks after $X$. fastidiosa inoculation. Results represent anthocyanin accumulation in each ecotype, normalized by subtraction of the basal anthocyanin content values of mock plants. The data are shown as the mean of 20 leaves. Bars represent standard errors. Different letters indicate statistical significance using a one-way analysis of variance, followed by a Tukey test $(P<0.05)$. Photographs on the right show representative leaves at 3 weeks after inoculation. $X f p=X$. fastidiosa subsp. pauca. planktonic phases, in which different molecules can be produced and recognized by the host and can trigger plant immunity responses (Rapicavoli et al. 2018b; Rodrigues et al. 2013; Zaini et al. 2018).

Compared with the other genotypes, the rap2.2 mutant showed a significantly higher anthocyanin accumulation in the presence of the bacterium (Fig. 5). This phenomenon seems to be correlated to the presence of RAP2.2 and its effects on the
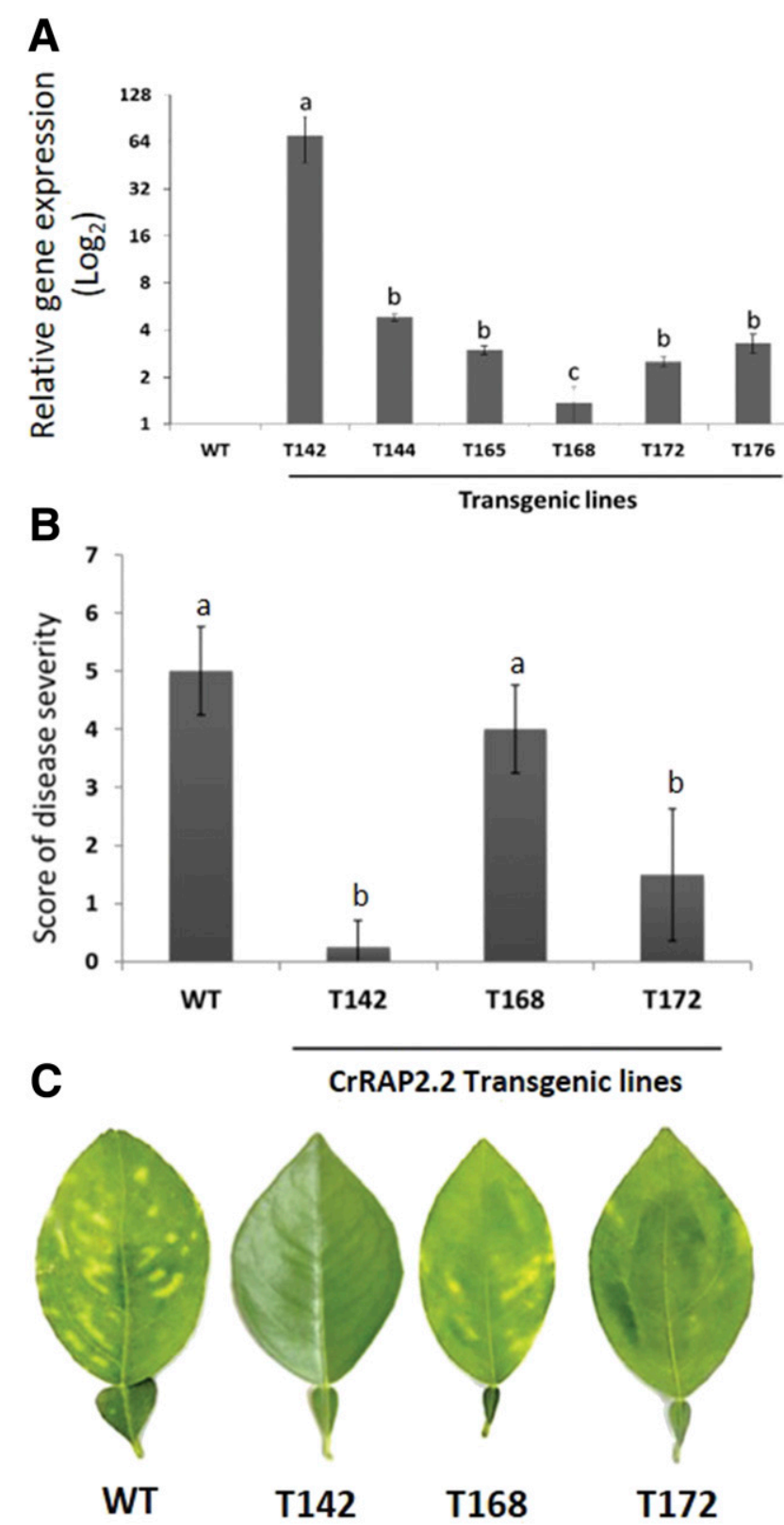

Fig. 6. Gene expression and symptom analysis of transgenic Citrus sinensis lines overexpressing CrRAP2.2. A, The relative expression of CrRAP2.2 was calculated in $C$. sinensis transgenic lines, with the gene expression level being normalized by the expression of $C s C Y C$ and $C s U B I Q$, which were used as endogenous controls. Results represent the mean of six replicates. $\mathbf{B}$, Severity of citrus variegated chlorosis (CVC) leaf symptoms in transgenic Pineapple sweet orange and wild-type plants was evaluated 18 months after Xylella fastidiosa inoculation. Disease severity was scored from 0 to 6 , where 0 represents no symptoms and 6 represents severe CVC symptoms. C, Representative leaves from wild type and transgenic lines 18 months after inoculation. Bars represent standard errors. Different letters indicate statistically significant differences among the means, which was calculated with a one-way analysis of variance followed by a Tukey test $(P<0.05)$. 
cells, since absence of AtRAP2.2 leads to a higher accumulation of anthocyanin and the phenotype can be restored by complementation using $C r R A P 2.2$. In addition, two copies of $R A P 2.2$ leads to a lack in anthocyanin accumulation in the presence of $X$. fastidiosa. Previous studies have shown that plant immune responses repress anthocyanin biosynthesis to activate plant defenses against bacterial or fungal pathogens (McLusky et al. 1999; Schenke et al. 2011; Serrano et al. 2012).

Our improved understanding of the function of $C r R A P 2.2$ in Arabidopsis led us to transform the $C$. sinensis Pineapple variety, which is highly susceptible to $X$. fastidiosa. Interestingly, protein alignment revealed that RAP2.2 from Pineapple variety (orange1.1g020408m from C. sinensis genome v1.0 [Joint Genome Institute]) lacks 63 amino acid residues at the $\mathrm{N}$ terminus of the protein, as compared with CrRAP2.2. In addition, 27 residues at this terminus are not shared by the proteins from these two species.

Transgenic lines with different expression levels of CrRAP2.2 allowed us to verify a negative correlation with CVC severity; we found that the higher the gene expression, the lower the disease symptoms (Fig. 6). Moreover, no change in development was observed in the transgenic plants (Supplementary Fig. S4B).

This is the first work in which a gene identified to be involved in $X$. fastidiosa defense response was functionally characterized using A. thaliana as a model plant. RAP2.2 has orthologous functions in $A$. thaliana and $C$. reticulata and proved interesting for developing transgenic varieties of $C$. sinensis resistant to CVC. As this gene also confers resistance to B. cinerea in A. thaliana (Zhao et al. 2012), it is possible that a broad-spectrum resistance to different pathogens might be attained in Citrus and other genera. This study opens new perspectives for using basic knowledge from a model plant for application in crops, accelerating the development of new varieties, especially for perennial plant species.

\section{MATERIALS AND METHODS}

\section{$X$. fastidiosa inoculation and}

\section{gene expression analysis in Citrus spp.}

A gene expression analysis was performed in two distinct experiments. First, the expression of RAP 2.2 was assessed in wild-type Citrus sinensis L. Osbeck (Pineapple variety) and Citrus reticulata Blanco (Ponkan variety) in response to $X$. fastidiosa. Briefly, X. fastidiosa subsp. pauca 9a5c was grown for 7 days in periwinkle (PW) solid media (Davis et al. 1981) at $28^{\circ} \mathrm{C}$. The cells were suspended in phosphate-buffered saline (PBS) to reach an optical density at $600 \mathrm{~nm}\left(\mathrm{OD}_{600}\right)$ of 0.3 (approximately $1 \times 10^{8} \mathrm{CFU} / \mathrm{ml}$ ) and were needleinoculated (Almeida et al. 2001) on the stem of $C$. sinensis and $C$. reticulata. Inoculations were performed on plants 6 months after grafting on Rangpur lime (Citrus limonia Osbeck). The xylem was collected at 1 and 7 days after inoculation and was submitted for RNA extraction according to Rodrigues et al. (2013). The second analysis was done in C. sinensis transgenic plants, aiming to evaluate $C r R A P 2.2$ expression.

Total RNA extraction from leaves was performed using a RNeasy plant mini kit (Qiagen, Hilden, Germany). The RNA integrity for the experiments was verified by electrophoresis on $1 \%$ agarose gel and the concentration was measured by NanoDropTM 8000 (Thermo Fisher Scientific, San Diego, CA, U.S.A.). The cDNA was synthetized in a $20 \mu \mathrm{l}$ total volume, from $1 \mu \mathrm{g}$ of total RNA, $500 \mathrm{ng}$ of oligo(dT) 15 , and $20 \mathrm{U}$ of RNAse inhibitor, according to the manufacturer instructions (GoScript Reverse Transcription System; Promega, Madison, WI, U.S.A.).
The RT-qPCR reactions were prepared in a final volume of $25 \mu$ with $10 \mu$ l of GoTaq qPCR master mix (Promega), 120 or $150 \mathrm{nM}$ of each gene-specific primer pair (Supplementary Table $\mathrm{S} 1$ ) and $3 \mu \mathrm{l}$ of 1:20 diluted cDNA synthetized from $1 \mu \mathrm{g}$ of total plant RNA. Amplifications were performed in an ABI 7500 Fast Real-Time PCR device (Applied Biosystems, Foster City, CA, U.S.A.), using the standard thermal profile: $95^{\circ} \mathrm{C}$ for $20 \mathrm{~s}$ followed by 40 cycles of $95^{\circ} \mathrm{C}$ for $3 \mathrm{~s}$, and $60^{\circ} \mathrm{C}$ for $30 \mathrm{~s}$. Each sample was analyzed in triplicate.

The primer efficiency and quantification cycle values were determined for individual RT-qPCR, using the algorithm of the Real-time PCR Miner (available online from the Stanford University). The relative mRNA level was calculated using the $2^{-\Delta \Delta \mathrm{CT}}$ cycle threshold method. A normalization factor (NF) for each sample was calculated by the geometric mean of the relative quantity $(\mathrm{RQ})$ values of the two reference genes: $U B I Q$ and $C Y C$ for $C$. sinensis Pineapple variety (Caserta et al. 2014, 2017). Normalized RQ (NRQ) of each sample was calculated as the ratio of the sample RQ and the appropriate NF. Individual fold change values were determined by dividing the sample NRQ by the mean NRQ of samples of the calibrator. This procedure renders a mean fold change value of 1 for the set of mock plants.

\section{In silico sequence analysis.}

CrRAP2.2 was one of the 81 genes, originally identified from an expressed sequence tag (EST) of Ponkan mandarin (Citrus reticulata Blanco), that were up-regulated when inoculated with $X$. fastidiosa (de Souza et al. 2007a). It is identical to the coding sequence Ciclev10025816m.g from C. clementina genome v1.0 available at the Citrus Genome Database. The CrRAP2.2 protein sequence was aligned with the $A$. thaliana genome available at The Arabidopsis Information Resource (TAIR) database, using BLASTP (available online from the National Institutes of Health) to search for putative orthologous sequences. The 3D models for the APETALA2 domains were obtained using the SWISS-MODEL software (available online from the Swiss Institute of Bioinformatics), which did the target-template alignment search against the SWISS-MODEL template library. The AtERF1 crystal structure (library 1 gccA) (Allen et al. 1998) was used as a template to obtain the 3D models. The alignments between AtERF1/AtRAP2.2 and AtERF1/CsRAP2.2 were obtained using PYMOL software (available online from the PyMOL Schrödinger technical support).

\section{CrRAP2.2 transcriptional activity assay.}

CrRAP2.2 was amplified from the A596p9ioGusi-FMV:: CrRAP2.2 vector (discussed below) (Supplementary Fig. S1), using primers with attB adaptors (Supplementary Table S1) and $P f u$ high fidelity DNA polymerase enzyme (Promega). It was purified in $1 \%$ agarose gel, using the Wizard SV gel and PCR clean-up system kit (Promega), was quantified using a Qubit 3.0 fluorometer (Invitrogen, Carlsbad, CA, U.S.A.), and was cloned into the entry vector using a BP Clonase II enzyme mix kit (Thermo Fisher Scientific). This entry vector has a 35SCrRAP2.2 cassette that was transferred to the pGILDA vector (Clontech, Mountain View, CA, U.S.A.) using the LR Clonase II enzyme mix kit (Thermo Fisher Scientific), resulting in an $\mathrm{AD}$ fusion construct, $\mathrm{AD}: \mathrm{CrRAP2}$.2. This construct was transformed into Saccharomyces cerevisae EGY48 (p8oplacZ), using the frozen-EZ yeast transformation II kit (Zymo Research, Irvine, CA, U.S.A.), to test whether this construct could activate the lacZ promoter. The LexA::CrRAP2.2expressing yeast strain was selected on SD-galactose inducing medium (BD Biosciences, San Jose, CA, U.S.A.) 
containing $80 \mu \mathrm{g}$ of $\mathrm{X}$-gal per milliliter, BU salts $(70 \%$ $\mathrm{Na}_{2} \mathrm{HPO}_{4} \cdot 7 \mathrm{H}_{2} \mathrm{O}$ and $30 \% \mathrm{NaH}_{2} \mathrm{PO}_{4}$ ), and -Ura/-His drop out supplement (BD Biosciences). Additionally, yeast strains were transformed with three truncated LexA::CrRAP2.2 constructs

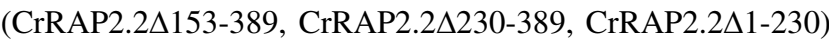
and were cloned following the same methods as for the whole CrRAP2.2 protein, to verify whether transcriptional activation signatures in the AtRAP2.2 (Welsch et al. 2007) corresponded to similar locations in the CrRAP2.2 protein sequence.

\section{CrRAP2.2 subcellular localization assay.}

Using the same procedures of the Gateway system (Hartley et al. 2000), the CrRAP2.2 amplicon was cloned into the destination vector $\mathrm{pB} 7 \mathrm{WGF} 2$ under the control of the cauliflower mosaic virus $35 \mathrm{~S}$ promoter (Karimi et al. 2002) to make the construct p35S-GFP::CrRAP2.2. This construct was transformed into Agrobacterium tumefaciens $\mathrm{C} 58 \mathrm{C} 1$ by the freezethaw method (Chen et al. 1994).

Subcellular localization of the GFP::CrRAP2.2 protein was determined by using a transient expression system (Walter et al. 2004). Agrobacterium strains carrying the constructs p35SGFP::CrRAP2.2 and p35S-GFP (pB7WGF2 empty vector) were infiltrated into leaves of four-week-old Nicotiana benthamiana plants. Initially, the $N$. benthamiana plants were grown in a greenhouse for 4 weeks and were then maintained in a growth chamber at $25^{\circ} \mathrm{C}, 60 \% \pm 5 \%$ relative humidity, and a 12 -h photoperiod with a light intensity of $100 \mu \mathrm{mol} \mathrm{m} \mathrm{m}^{-2} \mathrm{~s}^{-1}$. The leaves were marked around the infiltration site and, 3 to 5 days later, leaves were mounted onto microscope slides with $50 \%$ glycerol. Infiltration sites were visualized under a fluorescence microscope (Nikon Eclipse Ni), using specific filters for GFP (excitation:emission, 480/510 nm). Three independent experiments were performed.

\section{Arabidopsis genetic transformation and $X$. fastidiosa inoculation.}

The same constructs made for subcellular localization were also used for A. thaliana transformation. Employing the floral dip method (Clough and Bent 1998), Col-0 and rap2.2 plants were transformed with Agrobacterium tumefaciens C58C1 carrying p35S-GFP::CrRAP2.2 or p35S-GFP (pB7WGF2 empty vector). Transgenic lines were selected with BASTA $(0.0114 \%$ glufosinate ammonium) (BayerVR, Leverkusen, Germany) supplemented with $0.005 \%$ of Silwet L-77 (Lehle Seeds, Round Rock, TX, U.S.A.).

Seeds of Arabidopsis thaliana (L. Heyhn.) wild-type Columbia (Col-0, Arabidopsis Biological Resource Center [ABRC] stock CS60000), the rap2.2 mutant (ABRC stock SALK_010265C) with knockdown expression (Zhao et al. 2012), Col-0/CrRAP2.2 lines 1 and 5, and rap2.2/CrRAP2.2 lines 1 and 3 were sown on pots containing Multiplant substrate (Terra do Paraíso, Holambra, Brazil) with vermiculite in a 5:2 $(\mathrm{vol} / \mathrm{vol})$ proportion. Plants were grown in a Conviron growth chamber at $22^{\circ} \mathrm{C}, 60 \% \pm 5 \%$ relative humidity, and a $12-\mathrm{h}$ photoperiod with a light intensity of $100 \mu \mathrm{mol} \mathrm{m} \mathrm{m}^{-2} \mathrm{~s}^{-1}$.

Plant inoculation was performed according to the procedure described by Pereira et al. (2019). Briefly, four-week old plants were used for inoculation with $X$. fastidiosa subsp. pauca $9 \mathrm{a} 5 \mathrm{c}$, which was cultured at $28^{\circ} \mathrm{C}$ for 5 days on PW solid media (Davis et al. 1981), suspended in phosphate-buffered saline (PBS) buffer to reach an $\mathrm{OD}_{600}$ of 0.7 (approximately $10^{8}$ $\mathrm{CFU} / \mathrm{ml}$ ). To optimize inoculation efficiency, plants were not irrigated on1e day before and 1 day after inoculation. Four young, fully expanded rosette leaves were inoculated by dropping $5 \mu \mathrm{l}$ of the inoculum on the midrib at the petiole-leaf junction. The petiole tissue under the drop was pricked seven to eight times using a $13 \times 0.38 \mathrm{~mm}$ insulin needle (Becton
Dickinson, Franklin Lakes, NJ, U.S.A.). Water-inoculated plants were used as a mock control. No influence of the vector expression (pB7WGF2) on the pathogen response was verified (Supplementary Fig. S2). Plants were kept wellwatered during the experiment. Three independent experiments were performed.

\section{Evaluation of $X$. fastidiosa colonization of A. thaliana.}

Arabidopsis rosettes were harvested at 1,2, and 3 weeks after inoculation with $X$. fastidiosa, as described above, and were weighed to estimate the bacterial population per gram of tissue. qPCR reactions to quantify the pathogen were conducted in a total volume of $25 \mu \mathrm{l}$ containing $12.5 \mu \mathrm{l}$ of TaqMan Universal PCR master mix, $525 \mathrm{nM}$ of the primers CVC-1 (5'- GA TGA AAA CAA TCA TGC AAA-3') and CCSM-1 (5'-GCG CAT GCC AAG TCC ATA TTT-3'), 500 nM TAQCVC probe (5'(6FAM)AAC CGC AGC AGA AGC CGC TCA TC (TAMRA) p-3') (Oliveira et al. 2002), and 200 ng of DNA template. A negative (DNA from mock-inoculated plants) and a positive control (bacteria genomic DNA) were included in all experiments. The amplification parameters followed those described by Oliveira et al. (2002) $\left(50^{\circ} \mathrm{C}\right.$ for $2 \mathrm{~min}, 95^{\circ} \mathrm{C}$ for $10 \mathrm{~min}$, followed by 40 cycles of $15 \mathrm{~s}$ at $95^{\circ} \mathrm{C}$ and $1 \mathrm{~min}$ at $60^{\circ} \mathrm{C}$ ), and capture of the fluorescent signal was obtained in an $\mathrm{ABI}$ PRISM 7500 sequence detection system. All samples were processed in duplicates and the experiment was repeated at least twice (biological replicates). A standard curve for $X$. fastidiosa quantification was prepared following the methods described in Pereira et al. (2019).

\section{Anthocyanin quantification assay.}

Arabidopsis plants were inoculated as described and the procedure for quantifying the accumulated anthocyanin in response to $X$. fastidiosa was conducted according to Pereira et al. (2019). A total of 20 leaves were used to analyze the anthocyanin content in each ecotype inoculated or not (mock) with $X$. fastidiosa. The values obtained from mock plants (basal anthocyanin content) were subtracted from their respective inoculated ecotypes to determine the anthocyanin accumulation.

\section{Citrus transformation.}

The CrRAP2.2 sequence identified in the $C$. reticulata EST (identical to Ciclev10025816m.g) was chemically synthetized by the DNA Cloning Service company in a cassette containing the figwort mosaic virus (FMV) promoter. The cassette was inserted into the p9i-UbiAtm-oGusi vector to create the final construct A596p9ioGusi-FMV::CrRAP2.2. The p9i-UbiAtmoGusi vector has an optimized codon usage for plants and intron sequences inside the $\beta$-glucuronidase (GUS) reporter gene and kanamycin as a plant selection marker. The A596p9ioGusiFMV::CrRAP2.2 vector was used to transform shoots of C. sinensis, using an Agrobacterium-based method (Supplementary Fig. S1) (Caserta et al. 2014).

Seeds of sweet orange (C. sinensis) Pineapple variety were germinated in half-strength MS basal medium (Murashige and Skoog 1962) and were transformed as previously detailed (Caserta et al. 2014). To confirm transformation, a small piece of each transgenic shoot was excised and was incubated in $50 \mu \mathrm{l}$ of phosphate buffer containing 5-bromo-4- chloro-3-indolyl$\beta$-D-glucuronide for $16 \mathrm{~h}$ at $37^{\circ} \mathrm{C}$ to test GUS activity. In addition, genome integration of the transgene was evaluated by PCR using CrRAP2.2-specific primers (Supplementary Table S1). Ten buds from each shoot where transformation was confirmed were used for grafting onto Rangpur lime and were maintained in a mist chamber inside a greenhouse for acclimatization, to enable subsequent studies. GUS assays were 
performed for all leaves of propagated plants to identify any presence of chimeras. Only GUS-positive leaves from nonchimeric plants were used in the experiments. CrRAP2.2 expression was evaluated in confirmed transgenic plants as described above. Buds of each selected plant were multiplied into ten clones grafted onto Rangpur lime. GUS assays were performed using different parts of these new plants to detect any possible chimeras; only GUS-positive plants were used in screenings for $X$. fastidiosa resistance.

\section{Symptom analysis of transgenic citrus plants.}

Ten clones from three transgenic lines designated T142, T268, and T172, as well as ten clones from the wild type were used in symptom development assays. The presence of the bacterium was assessed by qPCR, as described above. Infected plants were assessed for the severity of CVC, were scored by three evaluators, using a diagrammatic scale (Amorim et al. 1993; Muranaka et al. 2013) and an image panel developed by Caserta et al. (2017).

\section{Statistical analysis.}

Statistical analyses were performed using Student's $t$ test $(P<0.05)$ or one-way analysis of variance followed by a Tukey's test $(P<0.05)$ in GraphPad Prism 8.2.1 for Windows (GraphPad Software, San Diego, CA, U.S.A.). All the experiments were performed at least three times with similar results, in which the data points represent the mean of three to 20 technical replicates, depending on the experiment.

\section{ACKNOWLEDGMENTS}

We thank L. F. C. da Silva from Centro de Citricultura "Sylvio Moreira"-Instituto Agronômico for greenhouse citrus propagation.

\section{AUTHOR-RECOMMENDED INTERNET RESOURCES}

Citrus Genome Database: https://www.citrusgenomedb.org The DNA Cloning Service website: www.dna-cloning.com TAIR database: https://www.Arabidopsis.org

\section{LITERATURE CITED}

Agüero, C. B., Uratsu, S. L., Greve, C., Powell, A. L., Labavitch, J. M., Meredith, C. P., and Dandekar, A. M. 2005. Evaluation of tolerance to Pierce's disease and Botrytis in transgenic plants of Vitis vinifera L. expressing the pear PGIP gene. Mol. Plant Pathol. 6:43-51.

Allen, M. D., Yamasaki, K., Ohme-Takagi, M., Tateno, M., and Suzuki, M. 1998. A novel mode of DNA recognition by a $\beta$-sheet revealed by the solution structure of the GCC-box binding domain in complex with DNA. EMBO J. 17:5484-5496.

Almeida, R. P. P., Pereira, E. F., Purcell, A. H., and Lopes, J. R. S. 2001. Multiplication and movement of a citrus strain of Xylella fastidiosa within sweet orange. Plant Dis. 85:382-386.

Amorim, L., Bergamin Filho, A., Palazzo, D., Bassanezi, R. B., Godoy, C. V., and Torres, G. A. M. 1993. Clorose variegada dos citros: Uma escala diagramática para avaliação da severidade da doença. Fitopatol. Bras. 18:174-180.

Caserta, R., Picchi, S. C., Takita, M. A., Tomaz, J. P., Pereira, W. E. L., Machado, M. A., Ionescu, M., Lindow, S., and de Souza, A. A. 2014. Expression of Xylella fastidiosa $\mathrm{RpfF}$ in citrus disrupts signaling in Xanthomonas citri subsp. citri and thereby its virulence. Mol. PlantMicrobe Interact. 27:1241-1252.

Caserta, R., Souza-Neto, R. R., Takita, M. A., Lindow, S. E., and de Souza, A. A. 2017. Ectopic expression of Xylella fastidiosa rpfF conferring production of diffusible signal factor in transgenic tobacco and citrus alters pathogen behavior and reduces disease severity. Mol. PlantMicrobe Interact. 30:866-875.

Chen, H., Nelson, R. S., and Sherwood, J. L. 1994. Enhanced recovery of transformants of Agrobacterium tumefaciens after freeze-thaw transformation and drug selection. Biotechniques 16:664-668, 670.
Choi, H. W., and Klessig, D. F. 2016. DAMPs, MAMPs, and NAMPs in plant innate immunity. BMC Plant Biol. 16:232.

Clough, S. J., and Bent, A. F. 1998. Floral dip: A simplified method for Agrobacterium-mediated transformation of Arabidopsis thaliana. Plant J. 16:735-743.

Coletta-Filho, H. D., Pereira, E. O., Souza, A. A., Takita, M. A., CristofaniYale, M., and Machado, M. A. 2007. Analysis of resistance to Xylella fastidiosa within a hybrid population of Pera sweet orange $\times$ Murcott tangor. Plant Pathol. 56:661-668.

Dandekar, A. M., Jacobson, A., Ibáñez, A. M., Gouran, H., Dolan, D. L., Agüero, C. B., Uratsu, S. L., Just, R., and Zaini, P. A. 2019. Trans-graft protection against Pierce's disease mediated by transgenic grapevine rootstocks. Front. Plant Sci. 10:84.

Davis, M. J., French, W. J., and Schaad, N. W. 1981. Axenic culture of the bacteria associated with phony disease of peach and plum leaf scald. Curr. Microbiol. 5:311-316.

de Souza, A. A., Takita, M. A., Coletta-Filho, H. D., Campos, M. A., Teixeira, J. E. C., Targon, M. L. P. N., Carlos, E. F., Ferraz, J. R., Fischer, C. N., and Machado, M. A. 2007a. Comparative analysis of differentially expressed sequence tags of sweet orange and mandarin infected with Xylella fastidiosa. Genet. Mol. Biol. 30 (3 Suppl):965-971.

de Souza, A. A., Takita, M. A., Coletta-Filho, H. D., Targon, M. L. P. N., Carlos, E. F., Locali-Fabris, E. C., Amaral, A. M., Freitas-Astúa, J., Silva-Pinhati, A. C. O., Boscariol-Camargo, R. L., Berger, I. J., Rodrigues, C. M., Reis, M. S., and Machado, M. A. 2007b. Analysis of expressed sequence tags from Citrus sinensis L Osbeck infected with Xylella fastidiosa. Genet. Mol. Biol. 30 (3 Suppl):957-964.

Giampetruzzi, A., Morelli, M., Saponari, M., Loconsole, G., Chiumenti, M., Boscia, D., Savino, V. N., Martelli, G. P., and Saldarelli, P. 2016. Transcriptome profiling of two olive cultivars in response to infection by the CoDiRO strain of Xylella fastidiosa subsp. pauca. BMC Genomics $17: 475$

Glazebrook, J. 2005. Contrasting mechanisms of defense against biotrophic and necrotrophic pathogens. Annu. Rev. Phytopathol. 43: 205-227.

Hartley, J. L., Temple, G. F., and Brasch, M. A. 2000. DNA cloning using in vitro site-specific recombination. Genome Res. 10:1788-1795.

Hinz, M., Wilson, I. W., Yang, J., Buerstenbinder, K., Llewellyn, D., Dennis, E. S., Sauter, M., and Dolferus, R. 2010. Arabidopsis RAP2.2: An ethylene response transcription factor that is important for hypoxia survival. Plant Physiol. 153:757-772.

Karimi, M., Inzé, D., and Depicker, A. 2002. GATEWAY vectors for Agrobacterium-mediated plant transformation. Trends Plant Sci. 7: 193-195.

Marcelletti, S., and Scortichini, M. 2016. Genome-wide comparison and taxonomic relatedness of multiple Xylella fastidiosa strains reveal the occurrence of three subspecies and a new Xylella species. Arch. Microbiol. 198:803-812.

Mauricio, F. N., Soratto, T. A. T., Diogo, J. A., Boscariol-Camargo, R. L., de Souza, A. A., Coletta-Filho, H. D., Silva, J. A. A., Medeiros, A. H. Machado, M. A., and Cristofani-Yaly, M. 2019. Analysis of defenserelated gene expression in citrus hybrids infected by Xylella fastidiosa. Phytopathology 109:301-306.

McLusky, S. R., Bennett, M. H., Beale, M. H., Lewis, M. J., Gaskin, P., and Mansfield, J. W. 1999. Cell wall alterations and localized accumulation of feruloyl-3'-methoxytyramine in onion epidermis at sites of attempted penetration by Botrytis allii are associated with actin polarisation, peroxidase activity and suppression of flavonoid biosynthesis. Plant J. 17:523-534.

Muranaka, L. S., Giorgiano, T. E., Takita, M. A., Forim, M. R., Silva, L. F., Coletta-Filho, H. D., Machado, M. A., and de Souza, A. A. 2013. Nacetylcysteine in agriculture, a novel use for an old molecule: Focus on controlling the plant-pathogen Xylella fastidiosa. PLoS One 8:e72937.

Murashige, T., and Skoog, F. 1962. A revised medium for rapid growth and bio-assays with tobacco tissue culture. Physiol. Plant. 15:473-497.

Niza, B., Coletta-Filho, H. D., Merfa, M. V., Takita, M. A., and de Souza, A. A. 2015. Differential colonization patterns of Xylella fastidiosa infecting citrus genotypes. Plant Pathol. 64:1259-1269.

Oliveira, A. C., Vallim, M. A., Semighini, C. P., Araújo, W. L., Goldman, G. H., and Machado, M. A. 2002. Quantification of Xylella fastidiosa from citrus trees by real-time polymerase chain reaction assay. Phytopathology 92:1048-1054.

Peña, L., Martín-Trillo, M., Juárez, J., Pina, J. A., Navarro, L., and Martínez-Zapater, J. M. 2001. Constitutive expression of Arabidopsis LEAFY or APETALA1 genes in citrus reduces their generation time. Nat. Biotechnol. 19:263-267.

Pereira, W. E. L., Ferreira, C. B., Caserta, R., Melotto, M., and de Souza, A. A. 2019. Xylella fastidiosa subsp. pauca and fastidiosa colonize 
Arabidopsis systemically and induce anthocyanin accumulation in infected leaves. Phytopathology 109:225-232.

Pérez-Donoso, A. G., Greve, L. C., Walton, J. H., Shackel, K. A., and Labavitch, J. M. 2007. Xylella fastidiosa infection and ethylene exposure result in xylem and water movement disruption in grapevine shoots. Plant Physiol. 143:1024-1036.

Rapicavoli, J., Ingel, B., Blanco-Ulate, B., Cantu, D., and Roper, C. 2018a. Xylella fastidiosa: An examination of a re-emerging plant pathogen. Mol. Plant Pathol. 19:786-800.

Rapicavoli, J. N., Blanco-Ulate, B., Muszyński, A., Figueroa-Balderas, R., Morales-Cruz, A., Azadi, P., Dobruchowska, J. M., Castro, C., Cantu, D., and Roper, M. C. 2018b. Lipopolysaccharide O-antigen delays plant innate immune recognition of Xylella fastidiosa. Nat. Commun. 9: 390.

Rodrigues, C. M., de Souza, A. A., Takita, M. A., Kishi, L. T., and Machado, M. A. 2013. RNA-Seq analysis of Citrus reticulata in the early stages of Xylella fastidiosa infection reveals auxin-related genes as a defense response. BMC Genomics 14:676.

Rogers, E. E. 2012. Evaluation of Arabidopsis thaliana as a model host for Xylella fastidiosa. Mol. Plant-Microbe Interact. 25:747-754.

Sabella, E., Luvisi, A., Aprile, A., Negro, C., Vergine, M., Nicolì, F., Miceli, A., and De Bellis, L. 2018. Xylella fastidiosa induces differential expression of lignification related-genes and lignin accumulation in tolerant olive trees cv. Leccino. J. Plant Physiol. 220:60-68.

Saponari, M., Boscia, D., Nigro, F., and Martelli, G. P. 2013. Identification of DNA sequences related to Xylella fastidiosa in oleander, almond and olive trees exhibiting leaf scorch symptoms in Apulia (Southern Italy). J. Plant Pathol. 94:688.
Schenke, D., Böttcher, C., and Scheel, D. 2011. Crosstalk between abiotic ultraviolet-B stress and biotic (flg22) stress signalling in Arabidopsis prevents flavonol accumulation in favor of pathogen defence compound production. Plant Cell Environ. 34:1849-1864.

Serrano, M., Kanehara, K., Torres, M., Yamada, K., Tintor, N., Kombrink, E., Schulze-Lefert, P., and Saijo, Y. 2012. Repression of sucrose/ultraviolet B light-induced flavonoid accumulation in microbe-associated molecular pattern-triggered immunity in Arabidopsis. Plant Physiol. 158:408-422.

Walter, M., Chaban, C., Schütze, K., Batistic, O., Weckermann, K., Näke, C., Blazevic, D., Grefen, C., Schumacher, K., Oecking, C., Harter, K., and Kudla, J. 2004. Visualization of protein interactions in living plant cells using bimolecular fluorescence complementation. Plant J. 40: 428-438.

Welsch, R., Maass, D., Voegel, T., Dellapenna, D., and Beyer, P. 2007. Transcription factor $R A P 2.2$ and its interacting partner SINAT2: Stable elements in the carotenogenesis of Arabidopsis leaves. Plant Physiol. 145:1073-1085.

Xu, K., Xu, X., Fukao, T., Canlas, P., Maghirang-Rodriguez, R., Heuer, S., Ismail, A. M., Bailey-Serres, J., Ronald, P. C., and Mackill, D. J. 2006. SublA is an ethylene-response-factor-like gene that confers submergence tolerance to rice. Nature 442:705-708.

Zaini, P. A., Nascimento, R., Gouran, H., Cantu, D., Chakraborty, S., Phu, M., Goulart, L. R., and Dandekar, A. M. 2018. Molecular profiling of Pierce's disease outlines the response circuitry of Vitis vinifera to Xylella fastidiosa infection. Front. Plant Sci. 9:771.

Zhao, Y., Wei, T., Yin, K. Q., Chen, Z., Gu, H., Qu, L. J., and Qin, G. 2012. Arabidopsis RAP2.2 plays an important role in plant resistance to Botrytis cinerea and ethylene responses. New Phytol. 195:450-460. 